\title{
Editorial: Old Problems and New Solutions in Pediatric Pulmonology
}

\author{
Sushil K. Kabra ${ }^{1}$ • Andrew Bush ${ }^{2}$
}

Received: 17 June 2015 / Accepted: 17 June 2015 / Published online: 25 July 2015

(C) Dr. K C Chaudhuri Foundation 2015

The series on pediatric pulmonology continues in this issue of the Journal, with four manuscripts that illustrate the new problems of our burgeoning speciality [1-4]. These are how the management of old problems (in this case TB) is being transformed by cutting edge science [1]; how the application of new technologies are creating challenging problems (congenital lung disease) [2]; and how new technologies are transforming the lives of children (neuromuscular disease and NIV) and taking mechanical ventilation, what used to be the prerogative of intensive care into the home, transforming the prognosis of children [3, 4]. However, as we shall see, the more the speciality advances, the more the basic truths we learned as students remain the bedrock of our practice.

Old disease, new technology: TB, the captain of the men of death, used to be simple - the tuberculin skin test, sputum (or other biological sample) microscopy and culture, and evidence based treatment with quadruple therapy for $2 \mathrm{mo}$, and rifampicin and isoniazid for a further 4 mo. Problem solved, even though diagnostics and determining sensitivities might take weeks - treatment could confidently be started while waiting. In children, admittedly diagnosis often had to be on clinical suspicion, because it was difficult to obtain samples.

Andrew Bush

A.Bush@rbht.nhs.uk

1 Division of Pediatric Pulmonology, Department of Pediatrics, All India Institute of Medical Sciences, New Delhi, India

2 Department of Pediatrics, Imperial College, London, UK; Department of Pediatric Respiratory Medicine, National Heart and Lung Institute, London, UK and Department of Pediatric Respiratory Medicine, Royal Brompton \& Harefield NHS Foundation Trust, London, UK
Enter the Dragon! Or rather, multidrug-resistant TB, extremely drug-resistant TB and totally drug resistant TB [5]. Perspectives have had to change rapidly; now we need rapid diagnosis and rapid sensitivity testing, for fear of starting drugs to which the organism is resistant, effectively but inadvertently proposing a single agent regime which will lead to worse problems of drug resistance. The problem has been made worse with the advent of HIV and the consequent worsening of the manifestations of TB. The modern approach is firstly to use novel tests to obtain specimens, such as sputum induction which can be taken in very young babies, and the string test. The second approach is newer microscopy and culture techniques, and specific molecular diagnostics, which are reviewed [1]. Molecular tests not merely detect MTB rapidly in sputum or gastric aspirates, but also allow drug sensitivities to be determined, a major management advance. The future will undoubtedly include molecular handprints on blood samples, as sophisticated techniques become cheaper and more accessible.

New technology, new problems: Increased monitoring of pregnant women with ultrasound scanning, and increasingly MRI, has identified abnormalities which previously passed us by. Congenital lung malformations come into just this category. In this issue, a simple clinical classification of congenital thoracic malformations (CTM), which is applicable pre- and post-natally, is described. However, description is one thing, evidence based management is another. How should you counsel a woman with a fetus with a large CTM at 20 wk of the pregnancy? If the baby is born well, what further imaging should be done and when? What is the risk of complications, and should well babies be submitted to surgery to reduce the risk? And when should surgery be performed? Finally, the biggest elephant in the room, what is the risk of primary lung malignancy in a baby with a CTM? High risk groups can be delineated, but most babies with CTMs are low risk. 
Worryingly, in a large Canadian study, CTMs which turned out to be or became a pleuropulmonary blastoma (admittedly a rare event) were indistinguishable from benign lesions [6]. And finally, even after complete resection of a CTM, the risk of malignancy elsewhere in the lungs remains. A real evidence free zone, and a challenge to young pulmonologists - can we get an evidence base by collaboration and the use of protocol driven assessment and follow up?

New technologies, transforming lives: The treatment of children with neuromuscular disease has undergone a sea change; in the last two decades, fatalism has been replaced by the realisation that these children can and do live full and satisfying lives, despite being wheelchair bound, with the use as appropriate of non-invasive mask ventilation during sleep to overcome inspiratory muscle failure, and airway clearance and assisted coughing to compensate for expiratory muscle dysfunction. The necessary aggressive respiratory management is outlined in an article in the Journal [3], including the diagnostic tools deployed to delineate the problem. The numerous diagnoses are summarised, some of which are presenting us with ethical dilemmas - what should be offered to a baby with severe SMA-1 with antenatal onset? Or a baby with the same diagnostic label, but presenting late and having preserved anti-gravity power in the arms, alert and interactive, and intellectually normal? There is also the diagnostic challenge to identify myopathies that may present with respiratory muscle dysfunction with well-preserved peripheral function. The management of nutrition, infection, and co-existent scoliosis is also challenging. There is no doubt that nasal mask ventilation has been transformative to these children [7]. Many of us are old enough to remember the days when it would have been inconceivable to ventilate a child in any way outside the Intensive Care unit. Now we are training families to have nasal ventilators at home, and other pieces of technology, and manage children with ever-more complex medical issues [4]. Selection of appropriate children is critical, as is the support of expert physiotherapists and respiratory nurses, with regular monitoring of ventilator settings. Prolonged home management, with greatly enhanced quality of life, is very feasible in such cases.

So these four articles highlight advances in our speciality, but one thing will always be true. The late, very great Max Klein was wont to say that "Empowerment is the most important principal in rehabilitation. Mother is the most sophisticated technology available in the world; love is of more consequence to the child than physical comfort. Intelligence is not related to literacy and best care is not necessarily more costly care" [8]. These words will continue to be cherished for as long as pediatricians look after children.

Conflict of Interest None.

Source of Funding None.

\section{References}

1. Triasih R. Newer diagnostic tests for pulmonary tuberculosis in children. Indian J Pediatr. 2015. doi:10.1007/s12098-015-1848-6.

2. Bush A. Rare lung diseases: congenital malformations. Indian $\mathbf{J}$ Pediatr. 2015. doi:10.1007/s12098-015-1800-9.

3. Khatwa UA, Dy FJ. Pulmonary manifestations of neuromuscular diseases. Indian J Pediatr. 2015. doi:10.1007/s12098-015-1814-3.

4. Preutthipan A. Home mechanical ventilation in children. Indian J Pediatr. 2015. doi:10.1007/s12098-015-1842-Z.

5. Udwadia ZF. MDR, XDR, TDR tuberculosis: ominous progression. Thorax. 2012;67:286-8.

6. Nasr A, Himidan S, Pastor AC, Taylor G, Kim PC. Is congenital cystic adenomatoid malformation a premalignant lesion for pleuropulmonaryblastoma? J Pediatr Surg. 2010;45:1086-9.

7. Chatwin M, Tan HL, Bush A, Rosenthal M, Simonds AK. Long term non-invasive ventilation in children: impact on survival and transition to adult care. PLoS One. 2015;10:e0125839.

8. Zar HJ, Weinberg EG. Max Klein: a great man and a great paediatrician. Thorax. 2015;70:396-7. 